\title{
The largest Swift AGN monitoring campaign: UV/optical variability in NGC 5548
}

\author{
S. D. Connolly*a, I. M. McHardy ${ }^{a}$, D. T. Cameron ${ }^{a}$, T. Dwelly ${ }^{b, a}$, P. Lira ${ }^{c}$, D. \\ Emmanoulopoulos $^{a}$, J. Gelbord ${ }^{d}$, E. Breedt ${ }^{e}$, P. Arevalo ${ }^{f, g}$, and P. Uttley ${ }^{h}$ \\ a Department of Physics and Astronomy, The University of Southampton, Southampton SO17 $1 B J$ \\ b Max-Planck-Institut für extraterrestrische Physik, Giessenbachstrasse 1, D-85748, Garching, \\ Germany \\ c Departmento de Astronomia, Universidad de Chile, Camino del Observatorio 1515, Santiago, \\ Chile
}

d Spectral Sciences Inc, 4 Fourth Avenue, Burlington, MA 01803 USA

e Department of Physics, University of Warwick, Coventry CV4 7AL

f Instituto de Astrofísica, Facultad de Física, Pontificia Universidad Católica de Chile, 306, Santiago 22, Chile

g Instituto de Física y Astronomía, Facultad de Ciencias, Universidad de Valparaíso, Gran Bretaña, 1111, Playa Ancha, Valparaíso, Chile

h Astronomical Institute 'Anton Pannekoek', University of Amsterdam, Science Park 904,

NL-1098 XH Amsterdam, the Netherlands

E-mail: imheastro.soton.ac.uk, sdclg08esoton.ac.uk

\begin{abstract}
We report on the largest Swift AGN monitoring program, concerning UV/optical variability in Seyferts. From 554 observations, over a 750d period, of the Seyfert galaxy NGC 5548, we see a good overall correlation between the X-ray and UV/optical bands, particularly on short timescales (tens of days) [1]. The UVOT bands are found to lag behind X-rays with a lag scaling as wavelength to the power $1.23+/-0.31$, in agreement with that expected (1.33) if UV/optical variability arises from reprocessing of X-rays by the accretion disc. However, the observed lags are $\sim 3$ times longer than expected from a standard Shakura-Sunyaev disc, raising real concerns about the detailed validity of this model. The results can be explained with a slightly larger mass and accretion rate, and a hotter disc, or if the disc is clumpy, thereby enhancing the emission from the outer regions.
\end{abstract}

Swift: 10 Years of Discovery,

2-5 December 2014

La Sapienza University, Rome, Italy

${ }^{*}$ Speaker. 


\section{Introduction}

The relationship between UV/optical variability and X-ray variability in AGN, and the origin of the UV/optical variability, is still not clear. Strong X-ray/UV or X-ray/optical correlations, with short lags of less than a day, have been observed on short timescales (weeks - months) by a number of studies [e.g. 2, 3, 4, 5, 6, 7, 8]. However, these studies show poorer correlations on longer timescales (months - years), usually due to long-term UV/optical trends not present in the X-ray variability, suggesting that UV/optical variability is dominated by different processes on different timescales. Reprocessing of X-rays into the UV/optical emission by the nearby accretion disc could cause an X-ray/UV correlation on short timescales, leading to UV/optical variations lagging the Xrays by the short light travel time between the X-ray source and the disc. Alternatively, if X-ray variability is produced by variability in the UV seed photon flux due to accretion variations in the disc at very small radii, a correlation could arise in which X-ray variability lags the UV-optical, by that same light travel time. Determining the precise lag between variations in the X-ray and UVoptical emission is therefore extremely useful in determining the origin of UV-optical variability.

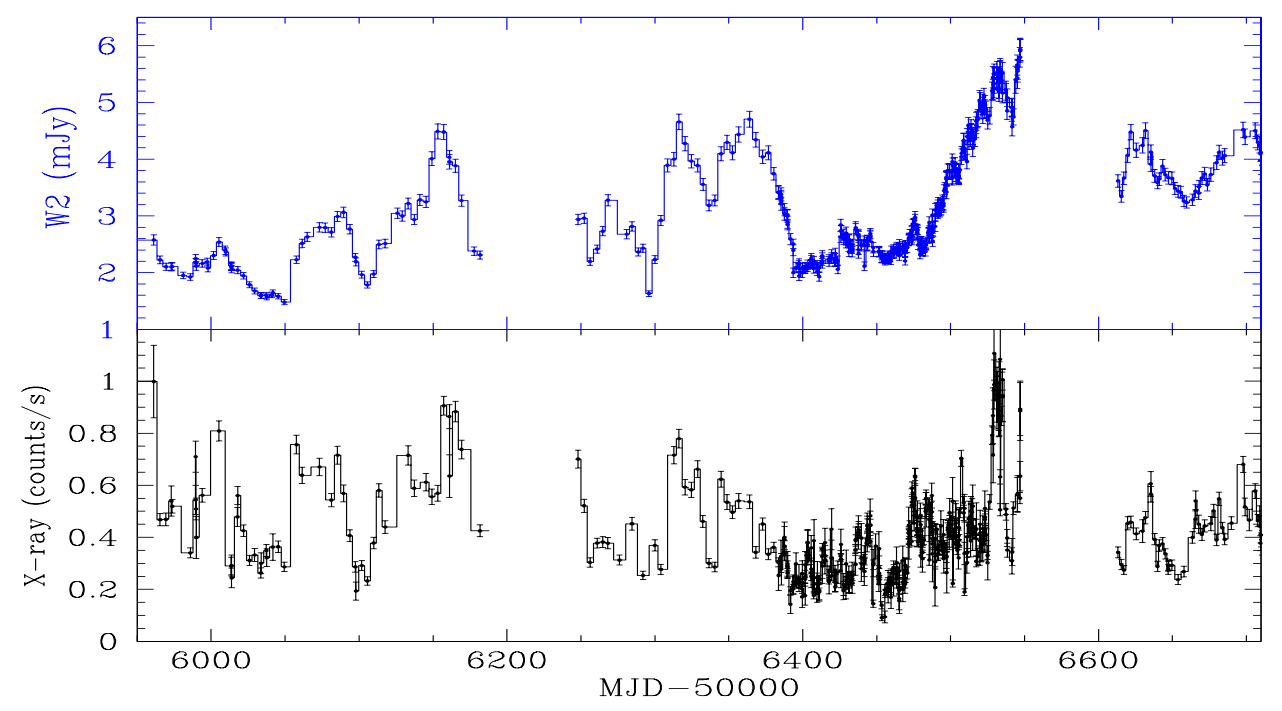

Figure 1: (Bottom panel) Long term Swift 0.5-10 keV X-ray count rate. (Top Panel) UVW2 flux.

Short ( $\sim 1$ d) lags of the X-rays by the optical are seen in all previous studies, which mostly consist of a combination of RXTE X-ray monitoring and ground-based optical monitoring. The lag has, however, never been measured well enough to completely rule out an optical lead unambiguously. Whereas ground-based monitoring is affected by weather, the Swift observatory can provide uninterrupted simultaneous X-ray and UV/optical monitoring, allowing more precise measurement of wavelength-dependent lags.

Using Swift observations, the B-band was shown to lag the X-rays by $<45$ mins in NGC 4395 [6] and interband lags were measured in NGC 2617 [8] which agree well with predictions from a reprocessing model.

Here, we discuss the largest Swift AGN monitoring campaign to date, of the Seyfert 1 galaxy NGC 5548, consisting of 554 observations over a 750d period [1]. The observations were not scheduled to follow particular events, but are instead typical of the long-term behaviour of NGC 


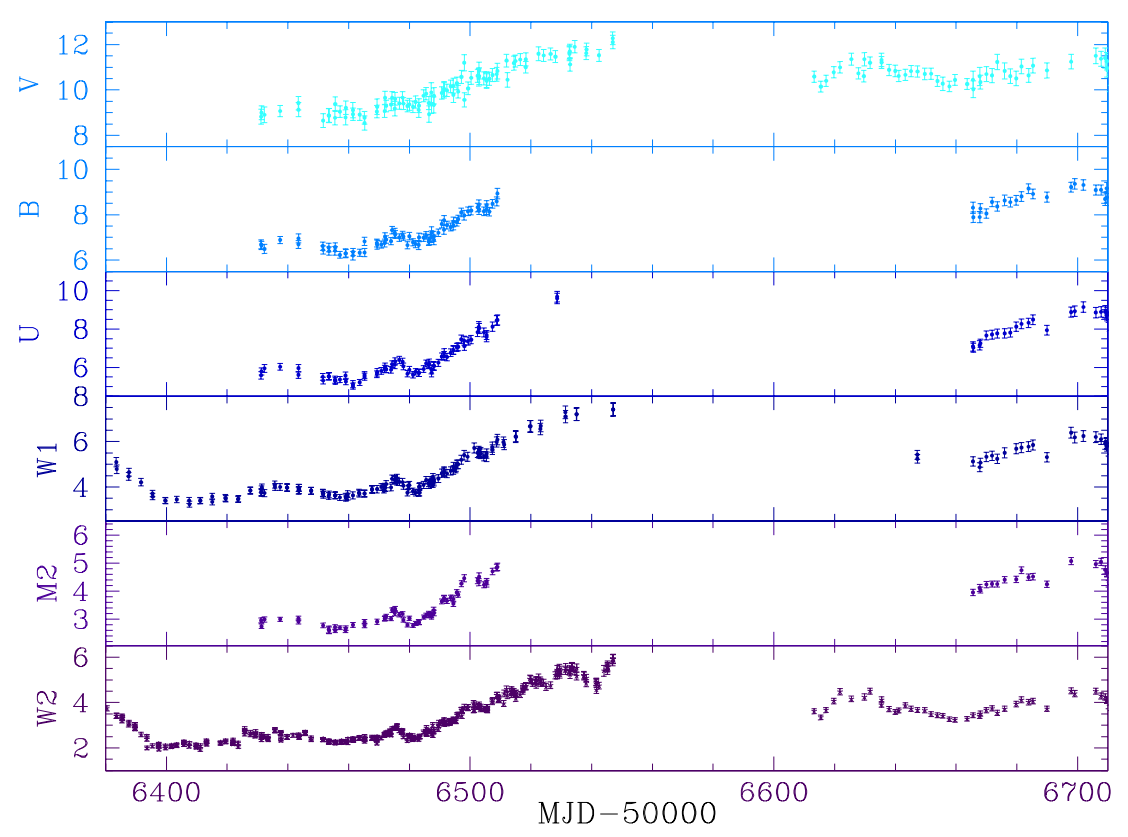

Figure 2: Multiband UVOT light curves in mJy.

5548. A strong X-ray/V-band correlation was already known to exist in NGC 5548 [2], but until this work the V-band lag had not yet been precisely defined.

\section{Observations \& Data Reduction}

The SWIFT X-ray data were taken by the X-ray Telescope (XRT, [9]) and the UV/optical observations taken by the UV and Optical Telescope (UVOT, [10]). All XRT observations were carried out in photon-counting (PC) mode and all UVOT observations were carried out in image mode. The data were analysed using our automatic pipeline, based upon the standard Swift analysis tasks, as described in e.g $[6,11]$. The X-ray data are corrected for any effects caused by vignetting and aperture losses and bad pixels. Drops in UVOT data points were investigated individually, and those which were due to bad tracking or bad pixels were removed.

Observations took place between MJD-50000 of 5960-6709, typically every 2 days, with some periods of less or more frequent sampling ( $1 \mathrm{~d}$ or $4 \mathrm{~d}$ ), and were mostly of $1 \mathrm{ks}$ though sometimes of 2 ks. Observations were usually split into 2 or more individual visits, improving time sampling. A total of 554 visits were made, giving, after rejection of bad data, 465 usable X-ray measurements.

As found previously between the X-ray and V bands [2], we find fairly close correspondence between the X-ray and UVW2 flux lightcurves. On short timescales $(\sim 10 \mathrm{~d})$, the correspondence is strong, but on longer timescales the amplitudes of variability are not always equal. Observations were made in additional UVOT filters from Day 6380; the resultant light curves are shown in Fig. 2. A very close correspondence is apparent between all UVOT bands.

\section{X-ray / UV-Optical Correlations}

\subsection{The X-ray / UVW2 lag}

In Fig. 3 we show the discrete [DCF, 12] and interpolation [ICCF, 13] cross-correlation functions (CCFs) of the UVW2 band, which is the best sampled of the UVOT bands, for the complete 

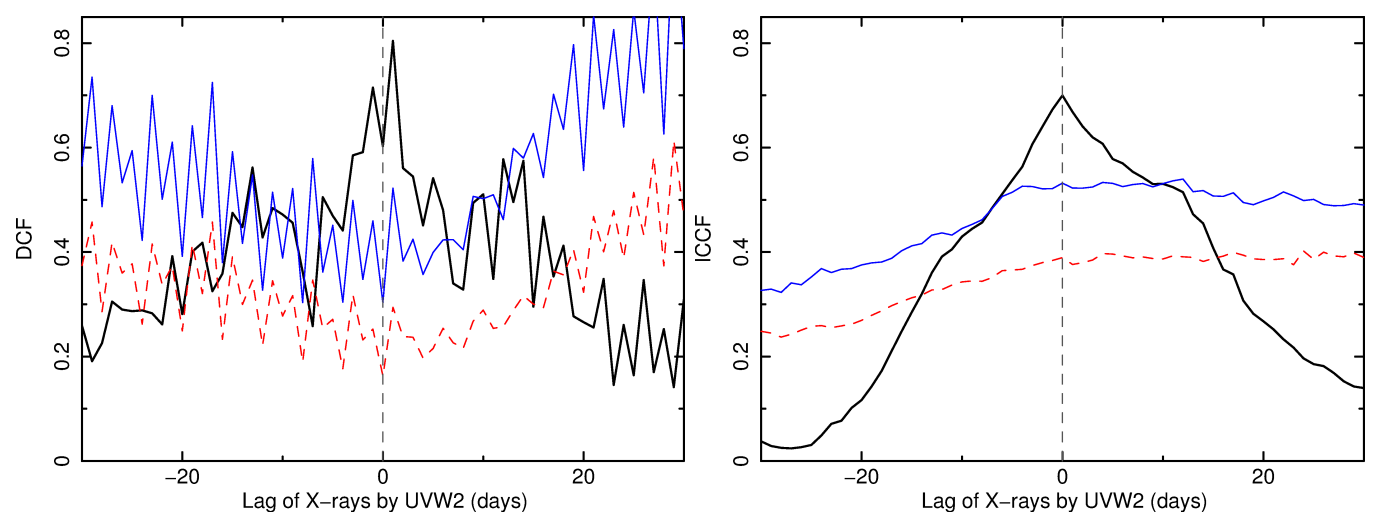

Figure 3: Left: Discrete cross correlation function between the X-ray and UVW2 lightcurves shown in Fig. 1. The $95 \%$ (dashed red) and $99.99 \%$ (solid thin blue) confidence levels are also shown. Right: Interpolation cross correlation function between the X-ray and UVW2 lightcurves shown in Fig. 1. The 95\% (dashed red) and 99\% (dashed blue) confidence levels are also shown.
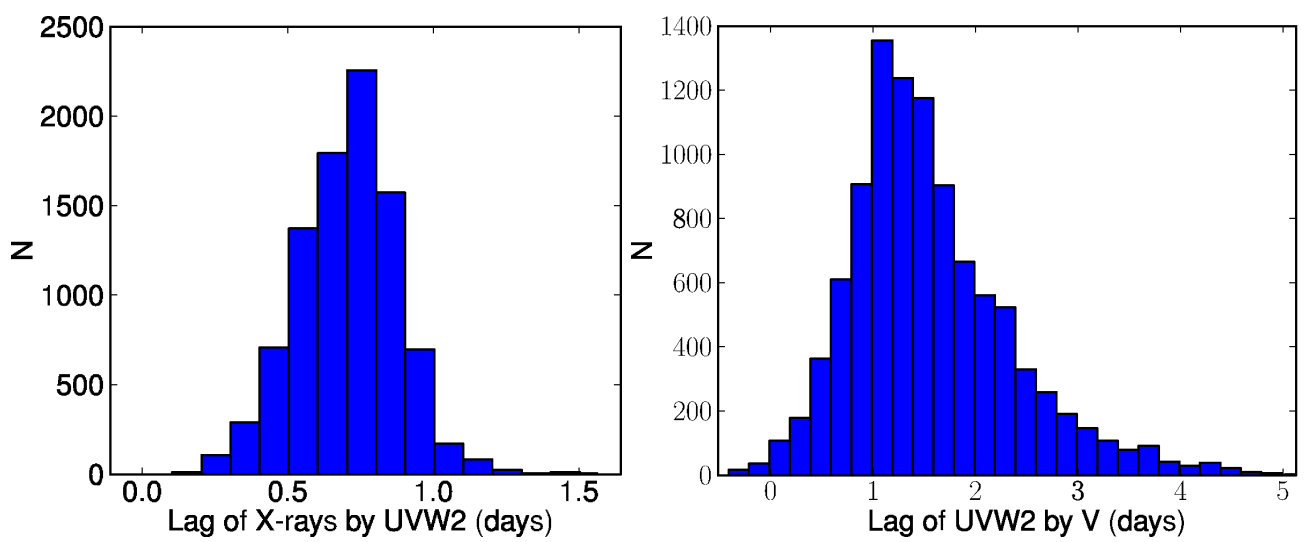

Figure 4: Lag distributions from Javelin: Left Panel UVW2 following X-rays using data, following mean subtraction, from the intensive period from 6383-6547. Right Panel V following UVW2 using data shown in Fig. 2.

X-ray and UVW2 datasets. Only the UVW2 band, being more slowly varying, is interpolated. The weighted mean ICCF of the 3 sections seen in Fig. 1 is taken, rather than interpolating across the gaps. The N\% confidence curves are also shown, and are defined such that (100-N)\% of correlations performed between the UVW2 data and randomly simulated X-ray lightcurves with the same variability properties as the X-ray data would exceed these levels (see e.g. [4],[14] for more details). Both the DCF and ICCF show a broad, but highly significant correlation, with a peak near a lag of zero, with the DCF favouring a lag of the X-rays by the UVW2 of about a day.

As correlation functions can be distorted by a long term variations in the mean level not present in both lightcurves, it is recommended practice to subtract a running mean [15]. A mean based on a running boxcar of width 20d is therefore subtracted from both UVW2 and X-ray light curves.

In order to refine the lag measurement, we calculated the distribution of lags from 10000 simulations for the period of intensive observation (Day 6383 - 6547), using the JAVELIN crosscorrelation program [16], used previously in e.g. [8, 17], allowing a lag range of \pm 10 d. JAVELIN assumes variability based on a damped random walk in both bands [18]; although the X-ray 


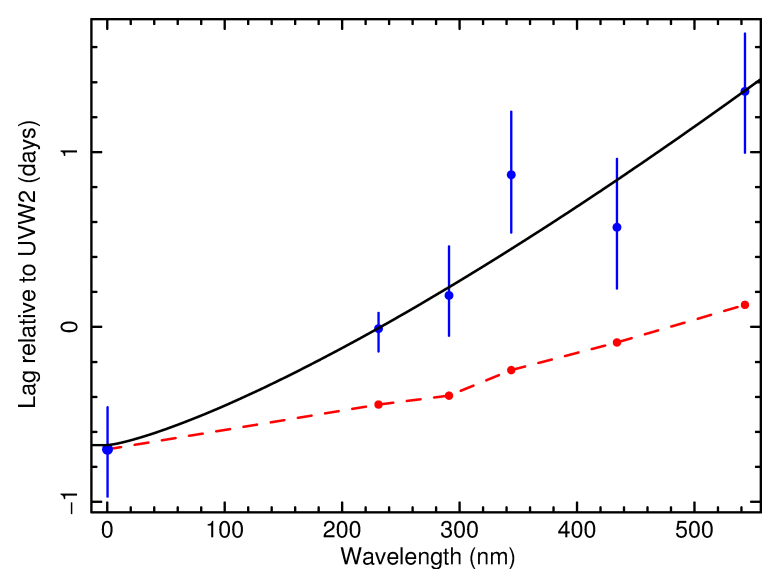

Figure 5: Lag of the X-ray and other UVOT bands relative to UVW2. Lag $\propto$ wavelength ${ }^{\beta}$ where $\beta=$ $1.23 \pm 0.31$. The lower, dashed, red line is the prediction for a standard disc as described in the text.

and UVW2 variability properties are slightly different, lag measurements are not significantly affected [8]. The resultant lag distribution, shown in Fig. 4 (left panel), has a median UVW2 lag of $+0.70_{-0.27}^{+0.24} \mathrm{~d}$, consistent with th lag found in both the discrete and the interpolative crosscorrelations. When applied to the non-mean subtracted light curves, JAVELIN does not converge to the same single distribution, presumably due to the uncorrelated long timescale variations.

\subsection{UVOT interband lags}

As the UVOT bands all show the same long term trends, it was not necessary to mean subtract the lightcurves. The lag distributions between UVW2 and the other UVOT bands were therefore calculated with JAVELIN using data shown in Fig. 2, an example of which is shown in Fig. 4, right panel. As some of the lag distributions are slightly asymmetric, use of the mode rather than the median would slightly reduce the lags in some cases, but the differences are small.

\subsection{X-ray reprocessing in a 'Standard Disc'}

On average, the UVOT bands lag the X-ray band with lags which increase with wavelength (Fig. 5). These lags can be compared to predictions of reprocessing from a simple accretion disc, for which the lag should vary as the $4 / 3$ power of wavelength [e.g. 19,5]. The lags were therefore fitted with a simple model of the form lag $=A+(B \times \lambda)^{\beta}$. The best fit, assuming Gaussian distributed errors, gives parameters of $A=-0.70 \pm 0.21, \beta=1.23 \pm 0.31$ and $B=3.2 \pm 0.6 \times 10^{-3}$, with a $\chi^{2}$ of 2.1 for 3 d.o.f. The slope, $\beta$, is similar to that derived by [8] for NGC 2617 (1.18 \pm 0.33$)$, but does not require an unphysical offset to the X-ray point, as they required.

This result demonstrates that the short term UV/optical variability in NGC5548 can be very well explained by reprocessing of X-ray emission from an accretion disc.

\section{Accretion disc Modelling}

In order to determine whether the observed lags can be explained by a disc consistent with other observed properties of NGC 5548, we model the disc using the methods described in [5] (c.f. [7]), including the effects of both X-ray and gravitational heating. The key parameters in the 
model are the X-ray heating, obtained by extrapolating the observed 2-10 keV luminosity $\left(L x_{2}-10\right)$ to $\sim 0.01-500 \mathrm{keV}$, the disc albedo, the height, $H$, of the $\mathrm{X}$-ray source above the disc (assuming a lamppost geometry), and the inner disc radius, $R_{i n}$.

We assume a black hole mass, $M_{B H}$, of $6.7 \times 10^{7} \mathrm{M}_{\odot}$ [20], an accretion rate in units of the Eddington limit, $\dot{m}_{E}$, of $\sim 0.03-0.04$ of Eddington [21], a high X-ray heating luminosity $6 \times L x_{2-10}$ (implying a low albedo of 20\%), $H=6 R_{g}$ (consistent with X-ray source sizes measured by other methods [22, 23]) and $R_{i n}=6 R_{g}$, the innermost stable circular orbit (ISCO) for a Schwarzschild black hole (where $R_{g}$ is one gravitational radius, equivalent to the Schwarzschild radius). This produces the dashed line shown in Fig. 5. The lags predicted from this standard disc model represent the time for half of the reprocessed light has been received, following impulse X-ray illumination. As the response is asymmetric, however, the peak response may be even faster, giving even shorter predicted model lags.

In order to increase the predicted lags from this homogeneous disc model such that they agree with observation, the geometry would have to be changed (e.g. $H=20 R_{g}, R_{i n}=20 R_{g}$ ), and a larger $\left.M_{B H}=10^{8} \mathrm{M}_{\odot}\right)$ and hotter $\left(\dot{m}_{E}=0.06\right)$ disc are required. In this case, disc flaring would have a smaller effect than an increased disc temperature.

\section{Discussion}

\subsection{Long X-ray/UV-Optical Lags}

The form of the observed wavelength-dependent lags, i.e. lag $\sim \lambda^{1.23}$ (Fig. 5), strongly support reprocessing of X-rays as the main cause of short-timescale UV/optical variability in AGN, however our observations also imply that this reprocessed emission originates further from the black hole than predicted by a 'standard' accretion disc model. The predictions and observations can only be reconciled by pushing parameter limits. It is important to note, however, that a larger than expected disc is also required [24] to explain microlensing observations, lending further support to the idea that the standard disc model is often inadequate, as proposed by e.g. [25]. The suggestion [24] of a low radiative efficiency is consistent with our requirement for $R_{i n} \geq 20$. Alternatively, an inhomogeneous disc, the outer portions of which contribute more flux than those of a uniform disc, would cause the disc to appear larger [26].

\subsection{Long-Timescale UVOT variability}

Although the correspondence of X-ray/UVW2 is very good on short timescales, and generally good on long timescales, from Day 6470 to 6547 there is a rise in the UV/optical which is significantly more pronounced than in X-rays. The UV/optical rise could be explained by an inwardly propagating rise in accretion rate, which eventually hits the X-ray emission region, however, the viscous timescales from $100 R_{g}$ are too long ( $\sim 10$ years, c.f. [4]). The data can therefore only be explained if the ratio of disc scale height to radius is larger than the value of 0.1 normally assumed, or the rise propagates through a corona over the disc rather than the disc itself, or the perturbation starts at a small radius $\left(\sim 20 R_{g}\right)$.

\subsection{Seed Photon Variations as a Driver of Variability}

Although the result that the X-rays always lead the UV/optical can be explained by assuming that all X-ray variability is generated within the corona, it is still interesting to ask why variations 
in seed photon flux, which would cause the UV-optical emission to lead, appear to have little affect on the measured lags. We suggest that the answer is a combination of relative solid angles and conservation of photons during the X-ray scattering process. A given single seed photon, Compton up-scattered into an X-ray photon with energies 10-100 $\times$ greater than the seed photon, can, through reprocessing in the disc, produce many UV-optical photons by black body emission. As the disc fills half the solid angle seen by the X-ray source, a large fraction of the X-ray photons will hit the disc. The total number of UV-optical photons produced in this way may therefore exceed the initial seed photon fluctuation. The reprocessing time, which is usually ignored in reprocessing calculations but which must be of finite length, could also add a further delay to the optical lightcurves, aiding agreement with observation.

\section{Acknowledgements}

This work was supported by STFC grant ST/J001600/1. SDC thanks the STFC for support under a studentship. PL acknowledges grant Fondecyt 1120328. JG gratefully acknowledges the support from NASA under award NNH13CH61C.

\section{References}

[1] McHardy, I. M. et al., Swift monitoring of NGC5548: X-ray reprocessing and short term UV/ optical variability, MNRAS, 444 (2014) 1469.

[2] Uttley, P., Edelson, R., McHardy, I. M., Peterson, B. M. and Markowitz, A., Correlated LongTerm Optical and X-Ray Variations in NGC 5548, ApJL, 584 (2003) L53.

[3] Arévalo, P. et al., Correlation and time delays of the X-ray and optical emission of the Seyfert Galaxy NGC 3783, MNRAS, 397 (2009) 2004.

[4] Breedt, E. et al., Long-term optical and X-ray variability of the Seyfert galaxy Markarian 79, MNRAS, 394 (2009) 427.

[5] Lira, P. et al., Optical and near-IR long-term monitoring of NGC 3783 and MR 2251-178: Evidence for variable near-IR emission from thin accretion discs, MNRAS, 415 (2011) 1290.

[6] Cameron, D. T. et al., Correlated X-ray/Ultraviolet/Optical Variability in the Very Low Mass AGN NGC 4395, MNRAS, 422 (2012) 902.

[7] Cameron, D. T., The Relationship between UV and Optical Variability and X-ray variability in Active Galactic Nuclei, PhD thesis, University of Southampton.

[8] Shappee, B. J. et al., The Man Behind the Curtain: X-rays Drive the UV through NIR Variability in the 2013 AGN Outburst in NGC 2617, ApJ, 788 (2013) 48.

[9] Burrows, David N., Hill, J. E., Nousek, J. A. and Kennea, J. A., The Swift X-ray Telescope, Sp. Sci. Rev., 120 (2005) 165.

[10] Roming, P. W. et al., The Swift Ultra-Violet/Optical Telescope, Sp. Sci. Rev., 120 (2005) 95. 
[11] Connolly, S. D., McHardy I. M. and Dwelly T., Long Term Wind-Driven X-Ray Spectral Variability of NGC 1365 with Swift, MNRAS, 440 (2014) 3503.

[12] Edelson, R. A. and Krolik, J. H., The Discrete Correlation Function: A New Method For Analyzing Unevenly Sampled Variability Data, ApJ, 333646.

[13] Gaskell, C. M. and Sparke, L. S., Line Variations in Quasars and Seyfert Galaxies, ApJ, 305 (1986) 175.

[14] Summons D. P., X-ray Power Spectral Densities of Active Galactic Nuclei, PhD thesis, University of Southampton.

[15] Welsh, W. F., On the Reliability of Cross Correlation Function Lag Determinations in Active Galactic Nuclei, PASP, 111 (1999) 1347.

[16] Zu, Y., Kochanek, C. S. and Peterson, B. M., An Alternative Approach to Measuring Reverberation Lags in Active Galactic Nuclei, ApJ, 735 (2011) 80.

[17] Pancoast, A., Brewer, B. J. and Treu T., Modeling reverberation mapping data I : improved geometric and dynamical models and comparison with cross-correlation results, MNRAS, 445 (2014) 3055 .

[18] Zu, Y., Kochanek, C. S., Kozłowski, S. and Udalski, A., Is Quasar Optical Variability a Damped Random Walk?, ApJ, 765 (2013) 106.

[19] Cackett, E. M., Horne, K., Winkler, H., Testing Thermal Reprocessing in Active Galactic Nuclei Accretion Discs, MNRAS, 380 (2007) 669.

[20] Bentz, M. C. et al., NGC 5548 in a Low-Luminosity State: Implications for the Broad-Line Region, ApJ, 662 (2007) 205.

[21] Vasudevan, R. V. et al., The power output of local obscured and unobscured AGN: crossing the absorption barrier with Swift/BAT and IRAS, MNRAS, 402 (2010) 1081.

[22] Chartas, G. et al., Revealing the Structure of an Accretion Disk Through Energy-Dependent X-Ray Microlensing, ApJ, 757 (2012) 137.

[23] Emmanoulopoulos, D., et al., General relativistic modelling of the negative reverberation X-ray time delays in AGN, MNRAS, 439 (2014) 3931.

[24] Morgan, C. W., Kochanek, C. S., Morgan, N. D. and Falco, E. E., The Quasar Accretion Disk Size-Black Hole Mass Relation, ApJ, 712 (2010) 1129.

[25] Antonucci, R., Active Galactic Nuclei and Quasars: Why Still a Puzzle after 50 years?, ArXiv, 1501.02001 (2015).

[26] Dexter, J. and Agol, E., Quasar Accretion Disks are Strongly Inhomogeneous, ApJL, 727 (2011) L24. 